\title{
MODELOS DE INTERVENCIÓN CON HOMBRES QUE EJERCEN VIOLENCIA EN LA PAREJA
}

\author{
JORGE CORSI \\ Universidad de Buenos Aires (Argentina)
}

\section{ANTECEDENTES}

La violencia hacia las mujeres por parte de sus parejas ha sido documentada, al menos en 42 países $^{1}$. En la mayoría de esos países se han desarrollado recursos legales, médicos y sociales para dar protección a las víctimas y a sus hijos e hijas.

Simultáneamente, se ha comprobado que las intervenciones dirigidas a los hombres que ejercen la violencia, ha recibido relativamente poca atención, tanto en los ámbitos gubernamentales como en los no gubernamentales.

Los programas de atención a los hombres que han ejercido violencia contra las mujeres surgieron a finales de la década del 70, en los Estados Unidos y Canadá. Su objetivo fue complementar los programas de atención y prevención de la violencia hacia las mujeres, teniendo en cuenta que la responsabilidad primaria de dicha violencia corresponde a quienes la ejercen. Desde un comienzo quedó claro que no se trataba de un tratamiento para una "enfermedad», sino de un proceso que procuraba la responsabilización frente a la violencia y el cambio en las relaciones abusivas hacia las mujeres.

Uno de los grupos pioneros en la implementación de estos programas fue $E M E R G E$, que introdujo la idea de la necesidad de partir de una base conceptual con perspectiva feminista si se quería ir hasta el fondo del problema. La diferen-

1. Adinkrah, M.: «Uxorcide in Fiji», Violence Against Women, 5:11 (1999), pp.1294-1320. GARCIA MORENO, C.: «Violence Against Women: International Perspectives», American Journal of Preventative Medicine, 19:4 (2000), pp. 330-333. Heise, L.; EllsberG, M. y GotTemoeller, M.: «Ending Violence Against Women", en Population Reports, Series L, n 11, Baltimore, Johns Hopkins University School of Public Health, Population Information Programme, 1999. HORNE, S.: "Domestic Violence in Russia», American Psychologist, 51:1 (1999), pp. 55-61. KozU, J.: «Domestic Violence in Japan", American Psychologist, 54:1 (1999), pp. 50-54. Subramaniam P. y SivaYoGan S.: "The prevalence and pattern of wife beating in the Trincomalee district in eastern Sri Lanka", Southeast Asian Journal of Tropical Medicine \& Public Health, 32:1 (2001), pp. 186-195. 
cia entre un programa meramente técnico y uno que adopte un punto de partida feminista es que el primero se limitará a la implementación de procedimientos tendentes al control de la conducta agresiva, mientras que el segundo supone una revisión profunda de las ideas sexistas y de los estereotipos de género que están en la base de las conductas de dominio y control sobre las mujeres.

La experiencia norteamericana y canadiense comenzó a ser imitada en otros países en la década siguiente, especialmente en Australia, los países escandinavos, Francia y Reino Unido. En países de habla hispana, el primero en contar con un programa específico para hombres fue Argentina donde, a partir de 1990, realicé una adaptación de los programas originalmente diseñados en un contexto anglosajón, para ser aplicados a poblaciones latinas. Luego siguieron México, Nicaragua y Costa Rica. En la actualidad, en todos estos países, la mayoría de las leyes que intentan dar una respuesta jurídica al problema de la violencia doméstica, incluyen el mandato judicial de tratamiento para los agresores.

\section{EL FORMATO DE LOS PROGRAMAS}

En la Conferencia sobre Violencia contra las Mujeres (Finlandia, 1999), se recogieron una serie de recomendaciones para las buenas prácticas en los programas dirigidos a los hombres que ejercen violencia en la pareja. En estas recomendaciones se hace referencia a los objetivos básicos que deben dirigir este tipo de programas, a la necesidad de justificarlos con rigor científico, a la no sustitución de medidas penales, a mecanismos que garanticen por encima de cualquier cosa la seguridad de las víctimas, a las fuentes de financiación y a la duración de estos programas, así como a la completa y amplia formación de los profesionales que trabajen en estos programas.

Por otra parte, hay distintas concepciones acerca de la etiología de la violencia masculina en la pareja ${ }^{2}$, que orientan las intervenciones en direcciones diferentes, según cual de ellas se adopte. Algunas de las hipótesis más frecuentes son:

1. Hipótesis cultural. El origen del maltrato a las mujeres por parte de los hombres se halla en los valores culturales patriarcales que lo favorecen, justifican o toleran como instrumento del mantenimiento del orden social y familiar.

2. Hipótesis estructural. Las raíces de la violencia residen en las desigualdades sociales y en la falta de oportunidades, que desencadenan en los individuos tensiones y agresividad, de la que acaban siendo víctimas sus cónyuges.

3. Hipótesis psicopatológica. El origen de la violencia masculina se halla en disfunciones de la personalidad como la impulsividad, la psicopatía o en el consumo abusivo de alcohol u otras drogas.

2. Wallace, H.: Family violence: Legal, Medical and Social Perspectivas, Boston, Allyn and Bacon, 1996. 
4. Hipótesis de la interacción. La etiología de la violencia en la pareja se halla en los estilos de relación verbal, de comportamiento y emocional que existen entre los cónyuges y, en general, en el seno de la familia.

5. Perspectiva jurídica. No constituye una explicación del maltrato sino que su propósito es incrementar el grado de detección, denuncia y condena penal de los maltratadores familiares.

La mayor o menor eficacia de este tipo de programas depende en gran medida de las bases éticas, ideológicas y teóricas en las que se apoye su diseño. Por lo tanto, resulta decisivo que el formato que adopten se ajuste al conocimiento específico del problema de la violencia de género. Algunos de los riesgos cuando se diseñan programas a la manera de tratamientos psicológicos convencionales son:

- La tendencia a psicopatologizar y por tanto a tratar psicopatológicamente el problema: La violencia de género en sus diversas manifestaciones hunde sus raíces en factores históricos, culturales, sociales, institucionales y familiares. La conducta violenta en el contexto doméstico debe ser entendida como un emergente de una compleja combinación de tales factores y no como el producto de alguna estructura psicopatológica. Las investigaciones en el campo de la Salud Mental han demostrado que es necesario invertir el razonamiento causal, para comprender que el maltrato y el abuso no sólo no son consecuencia de alteraciones psicopatológicas, sino que muy a menudo se constituyen en uno de los orígenes más frecuentes de dichas alteraciones. En tanto no hay patología a tratar, es conveniente pensar los programas como de reeducación, rehabilitación o de tratamiento psicosocial.

- El intento de abordar el problema desde los modelos psicoterapéuticos convencionales: Cuando el modelo teórico y metodológico, de cualquier orientación, se convierte en el eje del accionar terapéutico, sin un conocimiento adecuado de la especificidad del problema, se corre el riesgo de agravarlo. A menudo se termina culpabilizando a la víctima del maltrato o utilizando estrategias terapéuticas adecuadas para el tratamiento de conflictos familiares, pero absolutamente contraindicadas cuando de situaciones de violencia se trata.

- La no consideración de la variable género en el diagnóstico del problema: Las diversas manifestaciones de las situaciones de maltrato y abuso están atravesadas por dos variables indispensables para su comprensión: poder y género ${ }^{3}$. Cuando en un espacio terapéutico se desconoce el proceso de construcción de los géneros y su relación con las problemáticas emergentes de los vínculos abusivos, se corren serios riesgos de cometer errores terapéuticos que conduzcan a nuevas victimizaciones.

- Las nociones de "neutralidad", "secreto» y "privacidad»: Las situaciones de maltrato y abuso se pueden perpetuar en el tiempo gracias, precisamente, a la

3. BONINO, L.: «Violencia de género y prevención: el problema de la violencia masculina», en C. Ruiz Jarabo y P. Blanco (comp.): La violencia contra las mujeres. Prevención y detección, Madrid, Díaz de Santos, 2004, pp. 199-207. 
neutralidad de los otros y al secreto que garantiza la privacidad del hogar. Sólo comienzan a revertirse cuando una mirada externa las devela, las denuncia, no las legitima. Un contexto terapéutico de neutralidad, secreto y privacidad no hace sino reproducir simbólicamente las condiciones en las que la violencia encuentra su mejor caldo de cultivo

- Definición estilo "etiqueta» de las personas que utilizarán el programa. Habitualmente suele utilizarse el término maltratadores o violentos para definir a los hombres objeto de estos programas. Con esta definición, aun sin quererlo, se cataloga el problema como una cuestión de "ser», de identidad y por tanto de no responsabilidad propia. Hablar en cambio de «hombres que ejercen violencia», supone una definición en la que se incluye la violencia como algo, no de la identidad, sino del «hacer», del comportamiento, que como tal, podría no ejercerse, y supone responsabilización, a la que debe apuntar estos programas

Por lo tanto, a la hora de diseñar un programa de intervención con hombres que ejercen violencia contra las mujeres, es necesario:

$>$ Partir de un marco conceptual que incluya la perspectiva de género y la noción de maltrato como forma de ejercicio del poder masculino.

> Dar prioridad a la seguridad de la mujer, por sobre cualquier otro objetivo.

$>$ Integrar el programa a la red comunitaria de atención al problema de la violencia doméstica.

> Elaborar un formato específico, que lo diferencie claramente de los tratamientos psicológicos convencionales.

> Supervisar en forma permanente su evolución, así como la de sus profesionales, en intercomunicación con las organizaciones de ayuda a la mujer.

Una revisión de los programas de este tipo que existen alrededor del mundo fue realizada por Emily Rothman ${ }^{4}$, en el marco de una investigación encomendada por la Organización Mundial de la Salud. Después de haber hecho el relevamiento, describió cuales son los tópicos que caracterizan el trabajo de los grupos dirigidos a hombres que ejercen violencia en la pareja, tal como se puede ver en el siguiente cuadro:

4. ROTHMAN, E.; BUTCHART, A. y CERDÁ, M.: Intervening with Perpetrators of Intimate Partner violence: A Global Perspective, World Health Organization, Geneva (Switzerland), 2002. 


\begin{tabular}{|l|c|}
\hline \multicolumn{1}{|c|}{ Tópico } & $\begin{array}{c}\text { Proporción } \\
\text { en que los } \\
\text { Programas } \\
\text { utilizan el } \\
\text { tópico }\end{array}$ \\
\hline $\begin{array}{l}\text { Masculinidad: Discusión acerca del modo en que las normas culturales estereotipadas } \\
\text { acerca de lo masculino afectan el estilo que adoptan las relaciones intimas }\end{array}$ & $90 \%$ \\
\hline $\begin{array}{l}\text { Relaciones Intimas: Discusión acerca de las diferencias entre relaciones intimas } \\
\text { saludables y nocivas }\end{array}$ & $88 \%$ \\
\hline $\begin{array}{l}\text { Resolución de Conflictos: Métodos de resolución de problemas que no incluyan el uso } \\
\text { de la violencia }\end{array}$ & $86 \%$ \\
\hline $\begin{array}{l}\text { Tradiciones Culturales: Discusión acerca del modo en que la cultura patriarcal alienta } \\
\text { el uso de la violencia por parte de los hombres }\end{array}$ & $81 \%$ \\
\hline $\begin{array}{l}\text { Espiritualidad: Discusión acerca del modo que la fe y la dimensión espiritual afectan o } \\
\text { no a la tendencia hacia las conductas violentas }\end{array}$ & $76 \%$ \\
\hline $\begin{array}{l}\text { Control de la Ira: } \text { Técnicas para el control de las reacciones violentas frente a } \\
\text { situaciones que producen ira. }\end{array}$ & $76 \%$ \\
\hline $\begin{array}{l}\text { Habilidades para la paternidad: Entrenamiento para desarrollar una paternidad no } \\
\text { abusiva }\end{array}$ & $76 \%$ \\
\hline $\begin{array}{l}\text { Sanciones penales para quienes utilizan la violencia en las relaciones } \\
\text { intimas: Explicación de las leyes locales acerca de la violencia en la pareja. }\end{array}$ & $66 \%$ \\
\hline $\begin{array}{l}\text { Uso de Alcohol y de drogas: El efecto del alcohol y de las drogas como desinhibidores } \\
\text { de la conducta violenta. }\end{array}$ & $58 \%$ \\
\hline Trauma: Los efectos de experiencias traumáticas infantiles sobre la conducta adulta & $50 \%$ \\
\hline Estrés: Los efectos del estrés sobre la conducta. & $50 \%$ \\
\hline Salud sexual: Discusión acerca de las enfermedades de transmisión sexual en la pareja. & $44 \%$ \\
\hline $\begin{array}{l}\text { Opresión y discriminación: Discusión acerca de cómo el racismo, el clasismo, el } \\
\text { sexismo y otras formas de opresión y discriminación afectan la conducta }\end{array}$ & $44 \%$ \\
\hline $\begin{array}{l}\text { Organización comunitaria: Movilización para comprometer a otros a luchar contra la } \\
\text { violencia }\end{array}$ & $15 \%$ \\
\hline
\end{tabular}

Como se puede apreciar en este cuadro, el consenso más amplio está referido al hecho que la construcción de la masculinidad, en nuestra cultura, representa un verdadero factor de riesgo ${ }^{5}$, no sólo para la salud de los propios hombres, sino que sienta las bases de un desequilibrio de poderes que pone en riesgo la seguridad de las mujeres y de otros hombres ${ }^{6}$.

\section{ALGUNOS RESULTADOS}

En muchos de los países anteriormente mencionados, cuando son las administraciones públicas las que han buscado implementar estos programas, no siempre se han esperado resultados realistas: ninguno de estos programas puede garantizar la recuperación de hombres irrecuperables, la paz familiar sin separación, o la disminución de la necesidad de protección a las mujeres

5. BOnino, L.: «La violencia masculina en la pareja», en VVAA: Cárcel de amor, Madrid, Museo Nacional de Arte Reina Sofía, 2005, pp. 98-101.

6. CORSI, J. y BONINO, L.: "Violencia y Género: la construcción de la masculinidad como factor de riesgo", en J. Corsi y G. Peyrú: Violencias Sociales, Barcelona, Ariel, 2003, pp. 117-137. 
víctimas. Sólo pueden ser un complemento a las acciones para proteger a las mujeres, y no deben servir para crear falsas expectativas de seguridad para ellas o para des-responsabilizar a los poderes públicos de las acciones judiciales y preventivo-educativas necesarias para erradicar el problema.

En general, la motivación para el cambio es muy baja en los hombres que ejercen violencia contra las mujeres, pero la inclusión en un programa adecuadamente orientado suele incrementar la conciencia y el grado de responsabilización por su conducta, abriendo las puertas para comenzar a trabajar sobre su sistema de creencias sexistas, que es lo que realmente puede provocar el cambio.

Donde los hay, no todos los hombres que acuden a este tipo de programas se sienten motivados a seguirlo, y entre el 20 y el $60 \%$ de los casos que acuden por vez primera no lo hacen, especialmente si no hay sanción por ello. Este porcentaje disminuye si el programa está incluido en una red de recursos coordinados, donde tanto la derivación al programa como las deserciones puedan ser controladas

En los países en amplia experiencia en el tema, el criterio mínimo de éxito de estos programas se evalúa principalmente en función del abandono de la violencia física y de la violencia psicológica grave (especialmente el acoso e intimidación).

Desde este parámetro suele lograrse, con programas adecuados, un buen resultado entre el 30 y $60 \%$ de los casos que siguen el programa, excepto en el caso de los hombres violentos en general o psicópatas en el que el resultado sólo es positivo en el $5-10 \%$ de los casos. Se ha evaluado positivamente su utilidad para evitar reincidencias.

La disminución de la violencia psicológica y el aumento de los comportamientos respetuosos es de más difícil evaluación, pero si se toma en cuenta la sensación de seguridad y confianza de la mujer que fue victima del maltrato, los resultados son menos positivos, siendo mayores cuanto más duradero sea el programa (no menos de un año), y la consulta del hombre haya sido por cuenta propia (lo que se logra en programas ya muy conocidos por la comunidad).

El programa llevado a cabo en Buenos Aires desde el año $1990^{7}$ ha demostrado que la cantidad de hombres que consultan por voluntad propia aumenta a medida que es mayor el tiempo de permanencia y continuidad del servicio. En los años 1990 y 1991, el porcentaje de quienes concurrían espontáneamente, no llegaba al 1\% de los casos. Diez años más tarde, había llegado al $42 \%$. Esto significa que cuando un programa tiene continuidad, la comunidad lo incorpora dentro de su red de servicios y, al mismo tiempo, permite revisar el conocido mito de que los hombres no cambian ni quieren cambiar.

En primer lugar, he tenido que resolver un problema de terminologías, ya que la literatura especializada ha utilizado distintas denominaciones para aludir a los procedimientos que se llevan a cabo con maltratadores orientados a

7. La descripción del programa puede consultarse en CORSI, J. y otros: Violencia Masculina en la Pareja, Buenos Aires, Paidós, 1995. 
disminuir o eliminar su conducta violenta. He descartado el uso de las palabras "tratamiento" o "terapia», ya que ambas se refieren a las acciones que se emprenden con el objetivo de curar una enfermedad, y de ningún modo podemos considerar a la violencia masculina como tal, sino más bien como una conducta que, basada en un sistema de creencias sexista, tiende a asegurar el control y el dominio de la mujer como objeto de su posesión.

Tampoco me parecen adecuados los términos «rehabilitación» o «reeducación", ya que en ambos casos, se entendería que existen habilidades o aprendizajes que en algún momento han existido, que luego por algún motivo se han dañado, y nuestra tarea consiste en restaurarlos, del mismo modo que se rehabilita o se reeduca la motricidad de alguien que ha sufrido un accidente y presenta una secuela que dificulta la movilidad de sus miembros.

Al pensar en este tema, me he planteado que estas dificultades terminológicas se derivan de los intentos de psicologizar o medicalizar el problema de la violencia masculina hacia las mujeres, ignorando el fuerte componente cultural que tiene como subproducto de una cultura patriarcalmente construida.

En el pronóstico del potencial de cambio de los hombres que ejercen violencia, además de evaluar la demanda propia, hemos diseñado un instrumento que permite rápidamente diagnosticar a cual de los tipos descriptos por Donald Dutton $^{8}$ pertenece el consultante.

Cuando el perfil se corresponde con el Tipo B, el pronóstico es negativo, siendo la respuesta judicial la única posible. En cambio, los tipos A y C tienen un mayor potencial de cambio, dependiendo de que la intervención profesional sea la adecuada.

Se transcribe el mencionado instrumento de evaluación en el Apéndice de este artículo.

\section{CONCLUSIONES}

Después de haber evaluado alrededor de 2000 casos de maltratadores que concurrieron al programa de intervención psicoeducativa que he coordinado en la ciudad de Buenos Aires ( $y$ teniendo en cuenta que en Argentina la violencia en el ámbito doméstico no se halla penalizada, sino que se tramita en los juzgados civiles), algunas de las conclusiones que surgen de dicha evaluación pueden ser sintetizadas del siguiente modo:

- Los hombres que concurren a servicios de atención especializados en violencia masculina y lo hacen como resultado de la presión social o judicial, suelen presentar, en un primer momento, un incremento de los sentimientos hostiles y una tendencia a percibirse como "perjudicados» frente a lo que consideran una injusticia. Al tratarse de hombres que no están cumpliendo una condena sino que, como mucho, tienen una orden de alejamiento del hogar, esto representa un aumento del riesgo para la mujer, a quien perciben como la responsable de haberlo metido en problemas con su denuncia.

8. DutTon, D.: El golpeador, Buenos Aires, Paidós, 1997. 
- Con el transcurso del tiempo, se ha ido incrementando el porcentaje de hombres que concurren por propia voluntad a "pedir ayuda». Sin embargo, la mayoría de ellos está guiado por objetivos tales como «recuperar la pareja», «defender la unión de la familia», etc.

- Existe un alto porcentaje de la denominada «deserción inicial», es decir, aquellos hombres que cuando se les plantean los verdaderos objetivos que nos proponemos en el proceso de cambio, abandonan rápidamente el programa.

- Cuando se logran superar las etapas de los objetivos inaceptables y de las deserciones tempranas, el remanente de hombres que continúa en el programa requiere de un tiempo prolongado de trabajo psicoeducativo (nunca menos de un año) para comenzar a demostrar cambios.

- Los seguimientos realizados en los casos de hombres que han completado el programa, muestran que los mejores resultados los encontramos en aquellos que han comenzado nuevas parejas luego de completar su proceso. En cambio, el porcentaje de recaídas en los hombres que continúan con la misma mujer que en el pasado han maltratado, es mucho mayor.

Una primera conclusión que podemos extraer de esta síntesis es que las intervenciones con maltratadores, aún las específicamente diseñadas, tienen una eficacia restringida a un cierto porcentaje de hombres que, por sus características, resultan más permeables a la revisión en profundidad de sus sistemas sexistas de creencias, que están en la base de su conducta violenta. No obstante, seguimos pensando en la necesidad de incorporar las intervenciones dirigidas a los hombres, como parte integral de la estrategia de disminución de la tasa de violencia hacia las mujeres. Si tenemos en cuenta que la sanción judicial solamente alcanza a un pequeño porcentaje de hombres (aquellos que han cometido los actos de mayor gravedad), la mayor parte de la población de quienes ejercen diversos tipos de violencia hacia las mujeres queda fuera de todo tipo de intervención.

Hasta el momento, la mayor parte de los esfuerzos dirigidos a erradicar la violencia contra las mujeres estuvieron dirigidos a paliar las consecuencias de dicha violencia. El propósito de operar sobre las causas del problema nos lleva a distinguir entre las causas inmediatas (la conducta masculina que ocasiona el daño) y las causas estructurales del problema (la socialización masculina y femenina en el contexto de la cultura patriarcal). Mientras aguardamos que las estrategias de prevención a largo plazo produzcan cambios sustanciales en estas últimas, no podemos descuidar las acciones posibles para operar sobre las primeras. 


\section{APÉNDICE}

Tipología de hombres que ejercen violencia doméstica

\begin{tabular}{|l|l|l|}
\hline & SI & NO \\
\hline $\begin{array}{l}\text { 1. Los episodios de violencia son esporádicos } \\
\text { otra en privado) }\end{array}$ & & \\
\hline 3. La conducta violenta se produce exclusivamente en el contexto intimo & & \\
\hline 4. Tiende a minimizar su conducta violenta (quitarle importancia) & & \\
\hline 5. Utiliza justificaciones para su conducta violenta & & \\
\hline $\begin{array}{l}\text { 6. Recurre a la teoría de la provocación externa (la culpa la tiene la otra } \\
\text { persona) }\end{array}$ & & \\
\hline 7. Siente remordimientos después de cada episodio de violencia & & \\
\hline 8. Tiene dificultad para identificar y describir sus sentimientos & & \\
\hline 9. Presenta conductas celotípicas (se muestra celoso y controlador) & & \\
\hline 10. Tiene un humor cambiante (puede variar de un momento a otro) & & \\
\hline 11. Puede graduar su conducta violenta, de modo de no dejar huellas & & \\
\hline 12. Tiene antecedentes delictivos, penales o de conductas antisociales & & \\
\hline 13. No experimenta culpa después de los episodios violentos & & \\
\hline 14. No es capaz de comprender el sufrimiento de la otra persona & & \\
\hline 15. Tiene proyectos poco realistas para el futuro & & \\
\hline 16. Se resiste a analizar o discutir los problemas del pasado & & \\
\hline 17. También ejerce violencia con otras personas o en otros contextos & & \\
\hline 18. Calcula fríamente la utilización de su conducta violenta para dominar & & \\
\hline 19. Su objetivo principal es obtener sumisión y obediencia & & \\
\hline 20. Es minucioso, perfeccionista y dominante & & \\
\hline 21. Acumula tensiones sin reaccionar, hasta que explota & & \\
\hline 22. Frente a los conflictos, toma distancia o se cierra & & \\
\hline 23. Utiliza largos monólogos y técnicas de lavado de cerebro con la mujer & & \\
\hline 24. La critica, la humilla, pone a los hijos en su contra & & \\
\hline $\begin{array}{l}\text { 25. Tiene ideas rígidas acerca de división de roles, educación de los hijos, } \\
\text { etc }\end{array}$ & & \\
\hline $\begin{array}{l}\text { 26. Espera que la mujer se ajuste a las normas que él considera las } \\
\text { correctas }\end{array}$ & & \\
\hline $\begin{array}{l}\text { 27. Utiliza ataques verbales y/o supresión del apoyo emocional } \\
\text { 28. Se muestra colaborativo en la entrevista con un/a profesional }\end{array}$ & & \\
\hline
\end{tabular}

Referencias:

1-10: Tipo A (Perfil básico del maltratador)

11-18: Tipo B (Maltratador Psicopático)

19-28: Tipo C (Hipercontrolador)

Aclaraciones:

- No se trata de un cuestionario de auto-aplicación (las respuestas de los hombres a estos ítems no reflejarían la descripción real de sus conductas).

- Puede ser llenado a partir de una evaluación especializada y/o con la colaboración de la mujer. 
- Los 10 primeros ítems suelen ser comunes a los distintos tipos. Cuando no aparecen combinados con características de los otros tipos, constituyen el denominado «perfil básico del hombre que ejerce violencia doméstica».

- Para clasificar a un hombre dentro de los tipos B ó C, es necesario que, además de los rasgos pertenecientes al perfil básico, presenten el $50 \%$ de ítems positivos del tipo correspondiente. 